\title{
Calcification in a Recent Cerebral Infarct - Radiologic and Pathologic Correlation
}

\author{
J. Parisi, C. Place and S. Nag
}

\begin{abstract}
This 60 year old male developed a right hemiplegia and aphasia. A C.T. head scan showed a cerebral infarct which appeared hyperdense on a subsequent scan done 18 days after presentation. This was interpreted as indicating a hemorrhagic transformation resulting in discontinuation of anticoagulation therapy. At autopsy, the area of infarction in the left frontoparietal hemisphere appeared intensely green due to breakdown of the blood-brain barrier in the presence of jaundice. A striking finding on microscopy was the presence of calcium salts throughout the area of infarction but most prominent in the grey matter at the periphery of the infarct corresponding to the areas which appeared hyperdense on the CT head scan and stained intensely with bilirubin. There was no evidence of recent hemorrhage. This case illustrates that calcification can occur within weeks after the onset of a recent cerebral infarct and should be considered when interpreting the development of C.T. scan hyperdensity in recent cerebral infarcts.

RÉSUMÉ: Calcification au niveau d'un infarctus cérébral recent - corrélations radiologiques et anatomopathologiques Chez un homme âgé de 60 ayant développé une hémiplégie droite avec aphasie, un CT scan cérébral a montré un infarctus cérébral qui semblait hyperdense lors d'un scan subséquent fait 18 jours après l'accident cérébrovasculaire. Ce prénomène a été interprété comme indiquant une transformation hémorragique et conséquemment, l'anticoagulothérapie a été discontinuée.

A l'autopsie, la zone infarcisée dans l'hémisphère frontopariétal gauche semblait d'un vert intense, due à l'effondrement de la barrière hémo-encéphalique en présence d'ictère. A la microscopie, nous avons constaté une particularité frappante: la présence de sels de calcium dans toute la zone infarcisée, mais surtout dans la substance grise en périphérie de l'infarctus correspondant aux zones qui apparaissaient hyperdenses au CT scan cérébral et qui étaient colorées intensément par la bilirubine. Il n'y avait pas d'évidence d'hémorragie récente.

Ce cas illustre la possibilité que le processus de calcification puisse survenir en dedans de quelques semaines après un infarctus cérébral récent et devrait être considérée lors de l'interprétation d'une hyperdensité au CT scan, se développant sur un infarctus cérébral récent.
\end{abstract}

Can. J. Neurol. Sci. 1988; 15:152-155

Cerebral calcification has been observed in association with diverse lesions such as tumors, infections, and vascular malformations. Calcification of basal ganglionic vessels is a frequent finding in brains of elderly patients and has also been observed in association with disturbances of calcium and phosphate metabolism, in conditions such as hypoparathyroidism.

Calcification of cerebral infarcts is a very rare occurrence. A few case reports demonstrate hyperdensity in C.T. scans months to years after the presence of infarcts suggesting the presence of calcification. ${ }^{1.2}$ However, in none of these cases was a pathological follow-up available. In this case report the hyperdensity observed in a recent infarct on $\mathrm{C}$. T. scan 18 days after onset of symptoms was confirmed to be calcification by subsequent post-mortem studies.

\section{Case Report}

A 59 year old male with a past history of three myocardial infarcts underwent a triple coronary bypass operation. Twenty-one days later he presented with a three day history of cough and an acute onset of chest pain, dyspnea and hypoxemia. In the Emergency Room he rapidly developed a dense right hemiplegia and aphasia with a declining level of consciousness.

Physical examination revealed a blood pressure of $90 / 60 \mathrm{mmHg}$ pulse of $121 / \mathrm{min}$, respiratory rate of $33 / \mathrm{min}$, an elevated jugular venous pressure and a precordial auscultatory gallop. On chest examination, decreased air entry, coarse rales, and dullness to percussion were noted in the left base. Asymmetric right leg swelling was also present. Neurologic assessment showed hemiparesis, decreased corneal reflex and hemi-inattention on the right side and aphasia.

Laboratory studies demonstrated a $\mathrm{pH}$ of 7.53 , a $\mathrm{pO}_{2}$ of $46 \mathrm{mmHg}$ and a $\mathrm{PCO}_{2}$ of $21.7 \mathrm{mmHg}$ on room air. $A$ chest $x$-ray revealed a left 


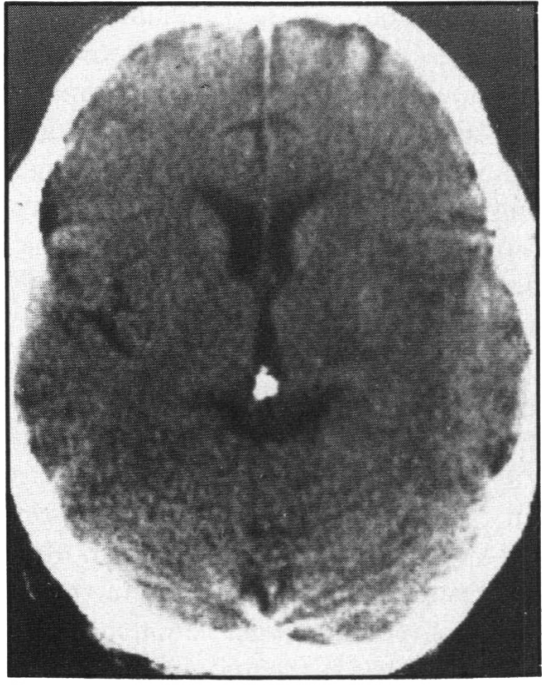

A

Figure 1 - Non-contrast C.T. scans of head (A) At 4 hours there is mild swelling of the left frontoparietal area with compression of the ipsilateral lateral ventricle.

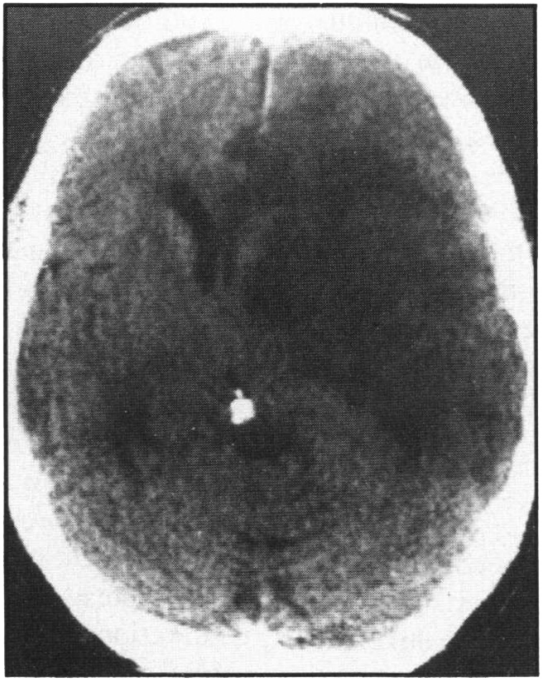

B

(B) At seven days a well-defined area of hypodensity is present in the region of the recent infarct. There is shift of structures across the midline with cingulate herniation and compression of the ipsilateral lateral ventricle.

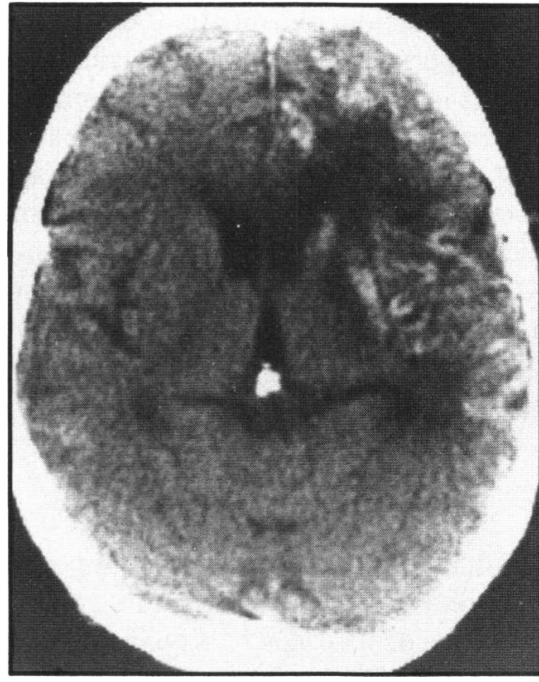

C

(C) At 18 days there is hyperdensity of the cortical ribbon, the caudate nucleus and putamen on the infarcted side. basal infiltrate and effusion while a radionuclide perfusion lung scan demonstrated multiple perfusion defects in radiographically normal lung which was interpreted as probable for pulmonary embolism.

A C.T. head scan done four hours after admission showed subtle changes suggestive of an extensive recent left frontoparietal infarction. The neurologic status continued to deteriorate and anticoagulant therapy was instituted to obtain a prothrombin time which was 1.25 times control values. Repeat radionuclide perfusion lung scan showed a changing pattern of defects. Anticoagulation and ventilatory support were continued but there was no improvement in neurologic function and a steady worsening of the patient's respiratory status. The I.C.U. course was complicated by superimposed sepsis, the onset of renal failure and intrahepatic cholestatic jaundice as manifested by mildly elevated transaminase (A.S.T. $35 \mathrm{U} / \mathrm{L}$, normal range less than $21 \mathrm{U} / \mathrm{L}$ ) and marked elevation of total bilirubin (total bilirubin $195 \mathrm{umol} / \mathrm{L}$, normal range less than $17 \mathrm{umol} / \mathrm{L}$ ). Alkaline phosphatase was normal and ultrasound examination excluded extrahepatic obstruction. Serum calcium levels also rose slightly $(2.95 \mathrm{mmol} / \mathrm{l}$, normal 2.15 to 2.65 $\mathrm{mmol} / \mathrm{l})$ three days prior to death.

Serial C.T. scans performed one and seven days after admission (Figures IA, B) confirmed the presence of a large frontoparietal cerebral infarction. However, the scan done eighteen days after admission showed striking hyperdensity of the basal ganglia and cortical ribbon in the infarcted region (Figure 1C). The possibility that the hyperdensity might represent calcification was not entertained and the hyperdensity was interpreted as hemorrhage into the infarct and anticoagulation was stopped.

In view of the continued deterioration and extensive cerebral damage further aggressive therapy was withheld and the patient expired 22 days after admission.

\section{AUTOPSY FINDINGS}

The cause of death was septicemia and bilateral acute bronchopneumonia with a left lower lobe abscess, due to Aerobacter aerugenes. In addition, a large saddle thromboembolus was present in the mainstems of both pulmonary arteries. The source of the emboli was the right femoral vein which showed a residual organising thrombus. Additional findings were old myocardial infarcts and chronic passive congestion of liver with cholestasis. Three small organising infarcts were observed in the spleen and kidneys.
The brain and attached dura weighed $1451 \mathrm{~g}$ prior to formalin fixation. The left frontoparietal region was soft and swollen in the area of the recent infarction. In this region, the gyri were flattened and sulci effaced and the cortex was green due to breakdown of the blood-brain barrier in the presence of hyperbilirubinemia.

On horizontal sectioning of the brain, the infarct was found to extend from the left frontal pole to the parieto-occipital junction in the area of distribution of the anterior and middle cerebral arteries (Figure 2A, B). Both the grey and white matter within the area of infarction showed bilirubin staining however green staining was more intense in the grey matter regions and this included the frontal, parietal, and temporal cortex, the anterior caudate nucleus, putamen, globus pallidus, claustrum, and insular cortex along the Sylvian fissure. The hyperdensity seen on CT scanning corresponded to the areas of intense green discoloration seen in the grey matter regions. There was no shift of midline structures nor was there evidence of recent or old hemorrhage in the area of infarction.

Microscopic sections of the brain were stained with hematoxylin-eosin and solochrome-cyanine. Sections from the left hemisphere revealed the typical histological features of a recent infarct. The centre of the infarct showed liquefaction necrosis with disruption of the neuropil and large numbers of foamy macrophages. There was preservation of the subpial rim of the molecular layer in the region of infarction. The surrounding white matter appeared pale using myelin stains and a marked gemistocytic response was present. In addition, vascular proliferation with endothelial hyperplasia was evident at the margins of the infarct. These features were consistent with an infarct of about three weeks duration as suggested by the history.

An additional and striking finding was the presence of amorphous calcium salts throughout the infarcted area but most prominent in the grey matter at the periphery of the infarct corresponding to the areas which appeared hyperdense on the CT head scan and stained intensely with bilirubin (Figure $3 \mathrm{~A}$, $B)$. The nature of the material was confirmed with a von Kossa 
stain for calcium phosphate. Calcium deposition was predominantly perivascular and no calcium was observed in vessel walls (Figure 4). Cytoplasmic calcium deposition allowed visualisation of dead neurons in the cortex and putamen.

The left internal carotid artery was completely occluded from the bifurcation to its termination into the left anterior and middle cerebral arteries by a recent thromboembolus (Figure 2B). The right internal carotid and remaining major cerebral arteries were patent revealing only patchy atherosclerosis. By microscopy the age of the thromboembolus corresponded with the age of the infarct.

\section{Discussion}

Calcification of cerebral infarcts is an exceedingly rare occurrence. The recent literature contains two reports describing a total of six cases. ${ }^{1,2}$ Kapila (1984) ${ }^{1}$ described the development of calcification in non-hemorrhagic infarcts of three patients as detected by serial C.T. scans which showed calcium nine, twelve, and forty-one months after the onset of infarction. None of the patients had elevated serum calcium levels. Kuzuhara et al. ${ }^{2}$ (1985), reported three patients in whom calcification was detected by C.T. scan two, five, and thirteen years after cerebral infarction. No calcification was noted on the initial C.T. head scans of these patients. These reports, however, describe only radiologic evidence of calcification and pathologic confirmation was not available. Furthermore, calcification in these

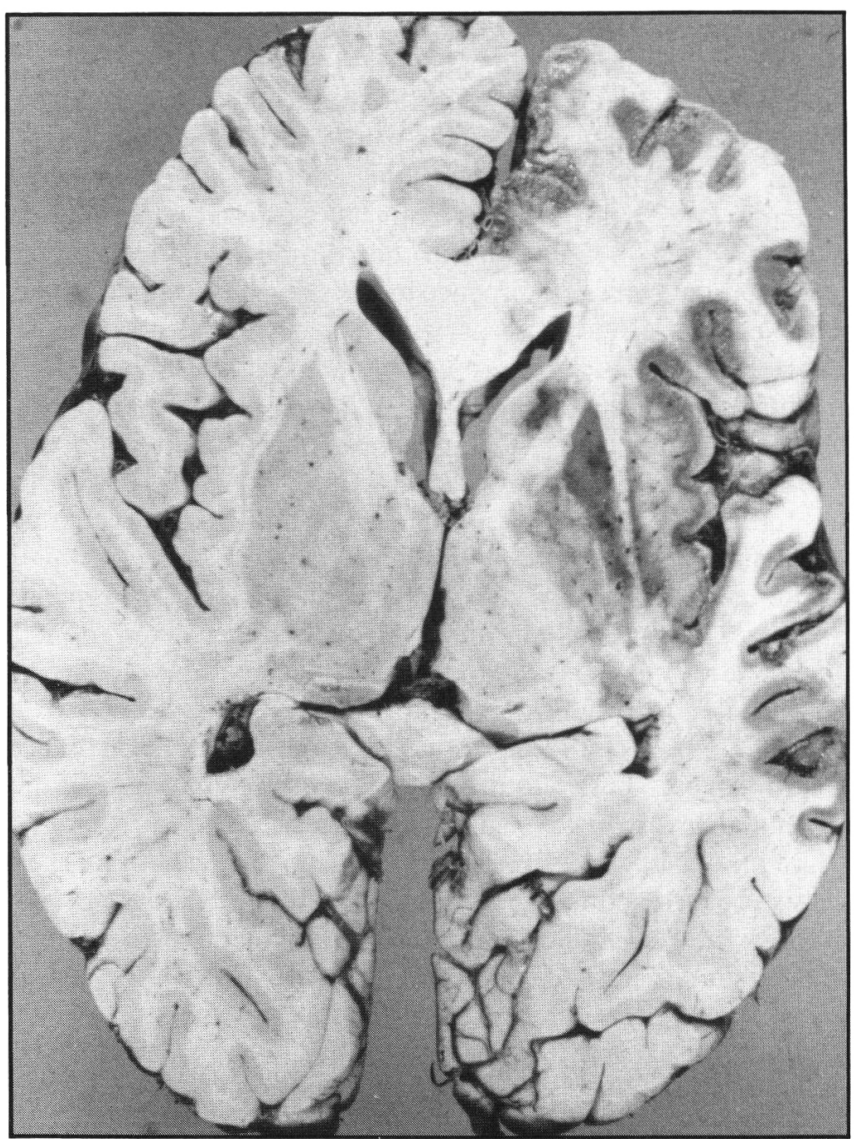

A

Figure 2 - Horizontal section of the brain (A) showing the area of recent infarction in the left fronto-parietal area. The dark grey discoloration of the grey matter in the area of infarction indicates the areas of green staining due to bilirubin extravasation. (B) The left internal carotid artery is occluded by a recent thromboembolus. cases occurred much later than in our patient. Calcium deposition occurring so early in the evolution of a cerebral infarct is most unusual and to our knowledge has not been previously described. A review of the last 150 cases of cerebral infarction at our institution revealed only one in which there was a large mass of unresorbed mummified tissue remaining in the centre of a massive ancient infarct. A post-mortem C.T. scan of this brain showed a hyperdensity at the rim of this necrotic tissue which on histology showed calcification.

In the present case, the initial interpretation of the C.T. scan hyperdensity was that of hemorrhage into the infarct. The risk of hemorrhage is known to increase with the size of the ischemic lesion and is higher if a mass effect is present or if there is a severe neurologic deficit. ${ }^{3}$ All of these correlating features were present in this case. A recent prospective study reported embolism as the cause of 29 percent of secondary hemorrhagic infarctions. ${ }^{3,4}$ Since our patient recently underwent major cardiac surgery, an embolic etiology was suspected. In addition he was anticoagulated in view of a suspected pulmonary embolism. Gross and microscopic examination, however, revealed no evidence of hemorrhage, and a post-mortem C.T. scan showed the same pattern of hyperdensity that was present preterminally.

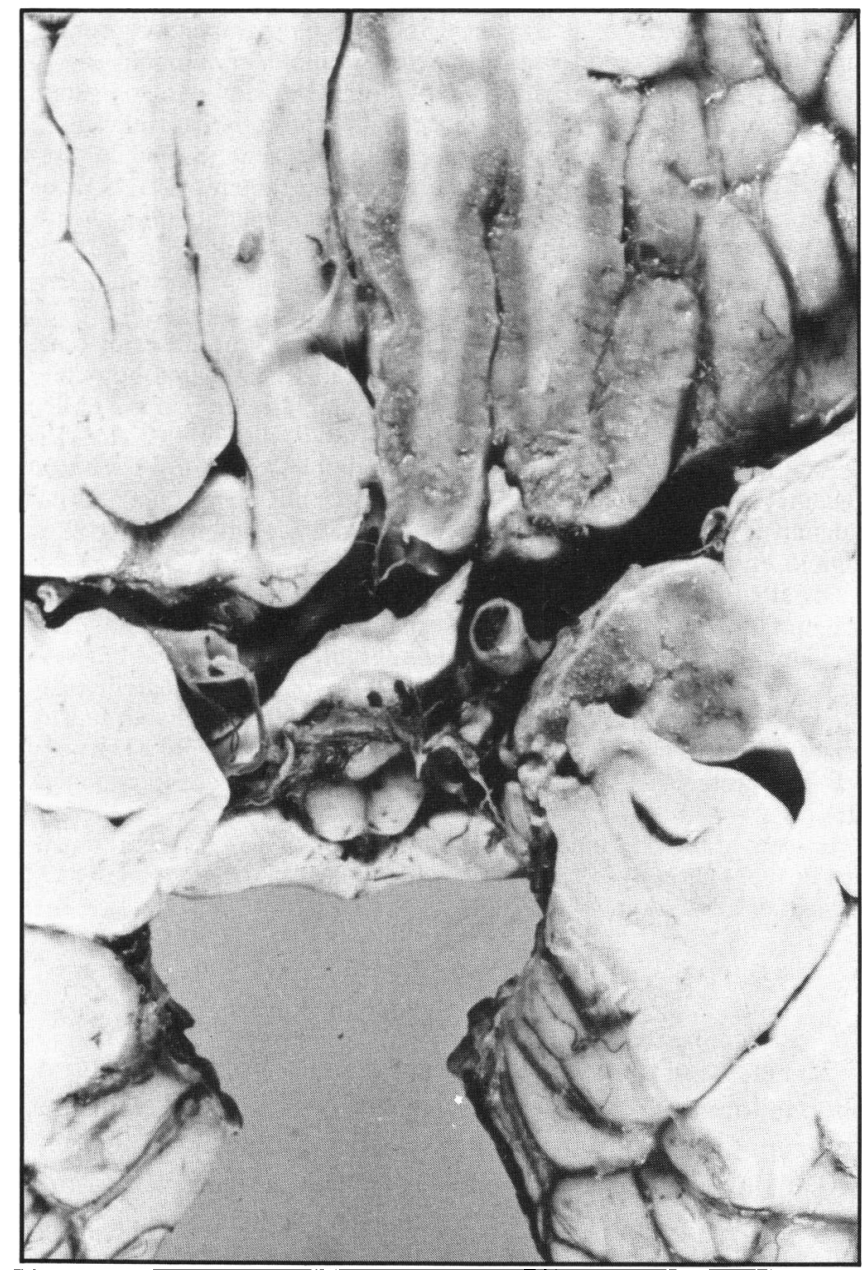



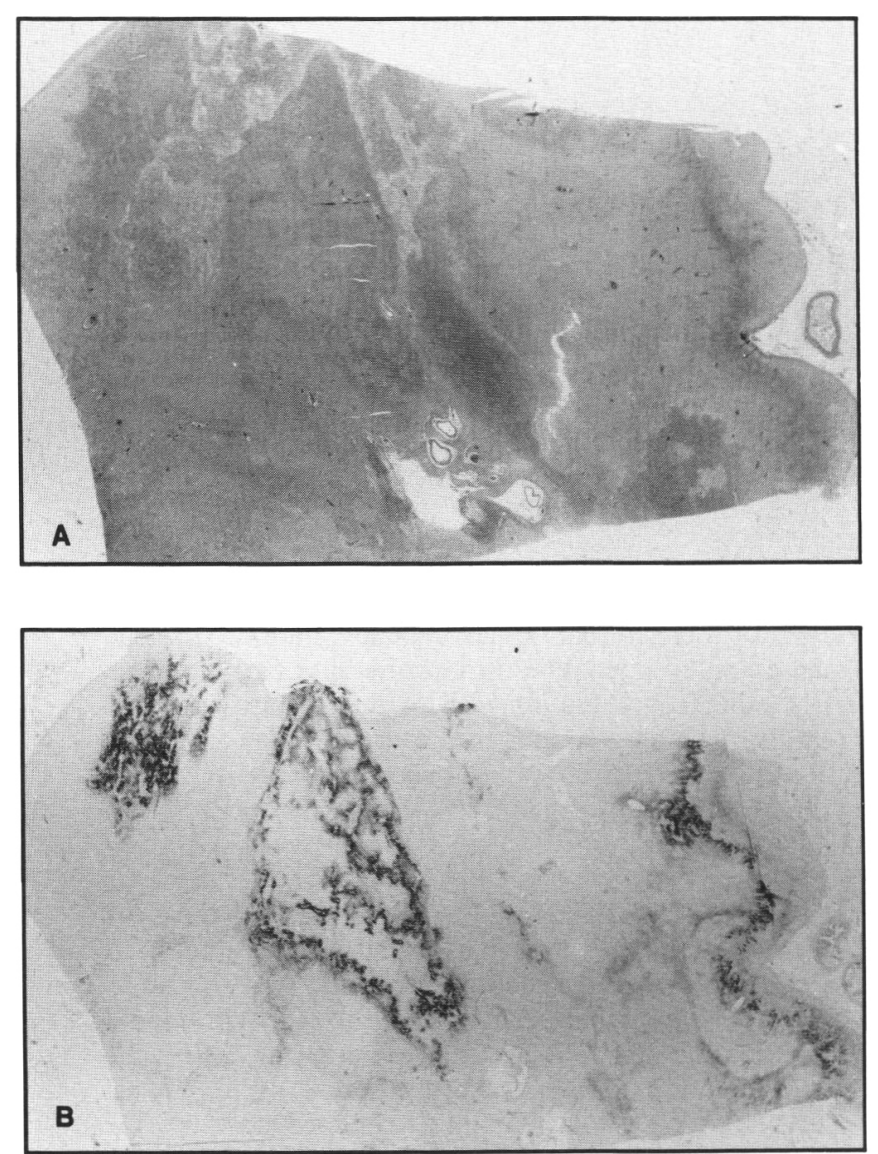

Figure $3-(A)$ Whole mount showing the area of infarction in the left basal ganglionic region. (B) An adjacent section stained with Von Kossa show's massive calcium phosphate deposition in the periphery of the infarct in grey matter structures such as the caudate, putamen and cerebral cortex. $A, B, \times 4.5$.

The observed green staining of the brain parenchyma is attributed to leakage of bilirubin across a damaged blood-brain barrier in the presence of jaundice. The distribution of calcium salts suggests the same mode of deposition along with serum protein leakage, but the exact pathophysiologic mechanisms are uncertain. The pattern is that of dystrophic calcification, having occurred in the absence of significant hypercalcemia. The serum calcium on a post-mortem blood sample was 1.80 $\mathrm{mmol} / \mathrm{L}$ and the highest recorded level during hospitalization was $2.95 \mathrm{mmol} / \mathrm{L}(2.15-2.65)$. Calcification was not found in any of the other body tissues including the small recent infarcts in the spleen and kidneys.

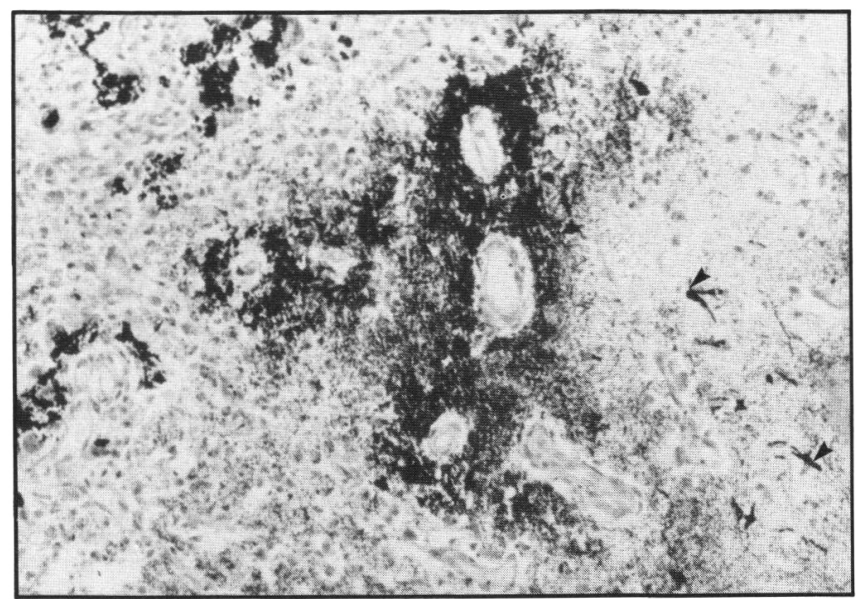

Figure 4-Microscopy of the left basal ganglionic region showing calcium phosphate deposition in neurons (arrowheads) and around the proliferated vessels at the margin of the infarct. $x 175$

Apart from the formation of pigment stones in the gall bladder calcium and bilirubin are not known to complex. They both, however, bind to plasma albumin and are considered to have been deposited independently of each other or in combination with albumin. The alcohol soluble bilirubin was washed out in the processing of the tissue sections and could not be demonstrated histologically. Clearly, as demonstrated by the previously reported cases, hyperbilirubinemia is not a requirement for the deposition of calcium. As well, a case on file at our institution with jaundice showed bilirubin staining of the infarct without development of calcification.

The importance of this case, however, lies in the rapidity of the onset of calcification. Recognition that this phenomenon could occur rapidly should be kept in mind if C.T. scan hyperdensity develops following recent infarction since the radiologist can by appropriate window settings differentiate between calcification and hemorrhage and thus assist with subsequent management decisions, particularly with regard to anticoagulation.

\section{REFERENCES}

1. Kapila A. Calcification of cerebral infarction. Radiology $1984 ; 153$ : 685-687.

2. Kuzuhara S, Naito Y, Namura Y, et al. C.T. demonstration of calcification within old cerebral infarcts. J Comput Assist Tomogr 1985; 9(2): 268-271.

3. Hornig CR, Dorndorf W, Agnoli AL. Hemorrhagic cerebral infarction - a prospective study. Stroke 1986; 17(2): 179-184.

4. Hakim AM, Ryden-Cooke A, Melanson D. Sequential computerized tomographic appearance of strokes. Stroke 1983; 14(6): 893-897. 